Supplementary Information 


\section{Instrumentation and Software}

The instrument consists of a fixed air-cooled micro-focus sealed Mo-K $\alpha(0.71073 \AA) \mathrm{X}$-ray source co-mounted with a doubly curved $\mathrm{Si}$ crystal monochromator and a fixed air-cooled Breeze CCD detector, Figure 1, which is housed in a $64 \mathrm{~cm}$ x $94 \mathrm{~cm}$ x $48 \mathrm{~cm}$ box weighing approx. $150 \mathrm{~kg}$, using mains power from a standard plug.

There is no requirement for either cooling water or a large electricity supply, and the instrument requires low maintenance. In addition, there is a port for a low temperature device, if desired. The detector is at a fixed distance of $39.3 \mathrm{~mm}$ from the sample, at $2 \theta=-$ $20.0^{\circ}$ and the goniometer is fixed with $\chi=35.1^{\circ}$. The system is free to move in $\omega$ and $\phi$. The instrument collects the data by three $\omega$ scans with a frame width of $0.5^{\circ}$, the first is a $180^{\circ}$ scan from $\omega=-20^{\circ}$ to $-200^{\circ}$, with $\phi=0^{\circ}$. The second and third $\omega$ scans also start at $\omega=-20$ ${ }^{\circ}$ and the scans are $180^{\circ} / 120^{\circ} / 60^{\circ}$, depending on the symmetry of the crystal, with $\phi=120$ ${ }^{\circ}$ for the $2^{\text {nd }}$ scan and $\phi=240^{\circ}$ for the third scan. The resolution limit of the instrument is $0.84 \AA$. The beam is slightly elliptical in nature, with a wider profile in the horizontal direction, to allow for a degree of misalignment, as discussed later in Section 3. The beamstop is directly attached to the detector face, which allows for ready visualization of the source since it filters out most, but not all, of the direct beam. Figure 2 shows a representative diffraction pattern, in this case for compound 2, discussed later in Section 4.
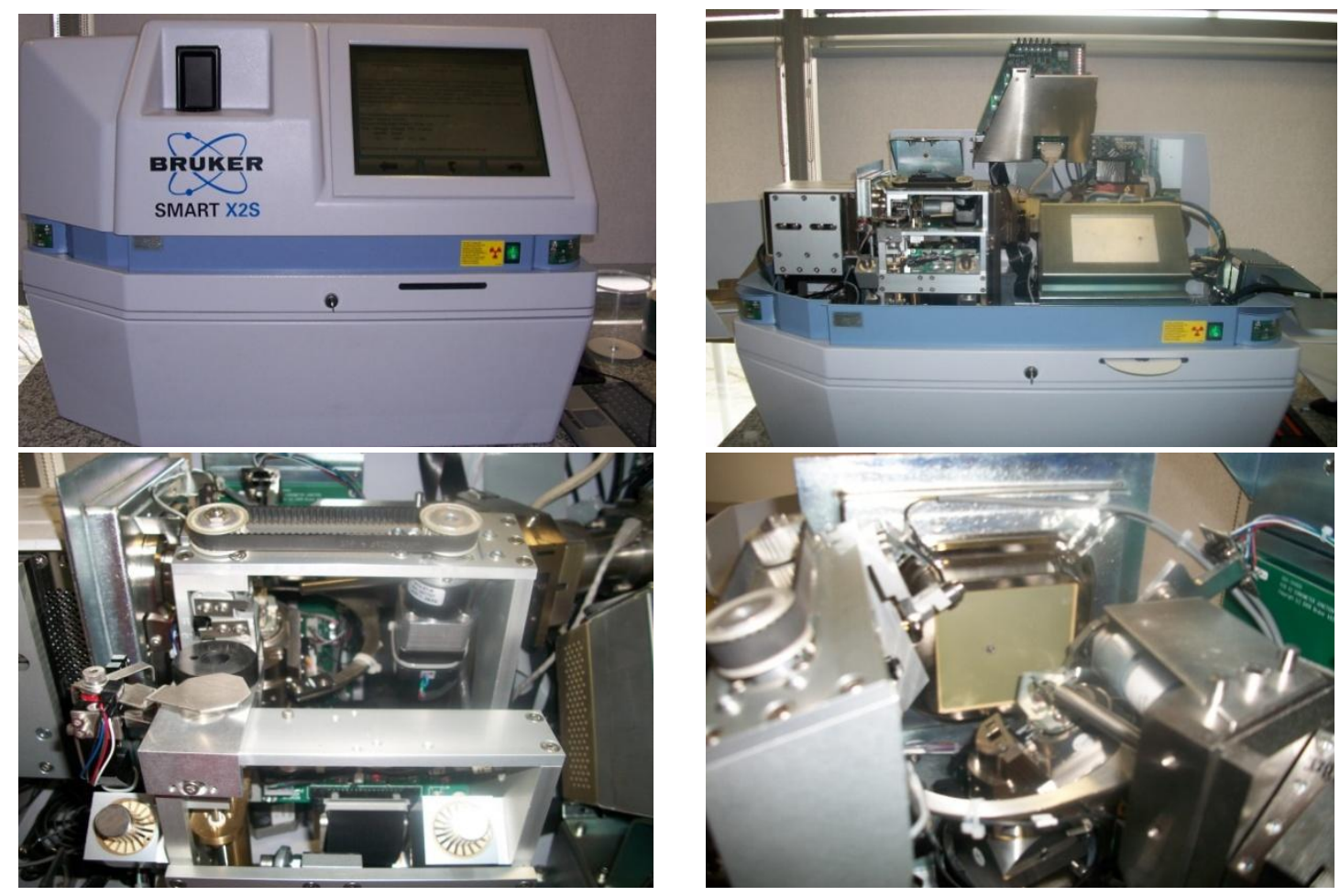

Figure 1: The Benchtop Bruker SMART X2S: external view (top left), cover removed (top right), patented sample loader (bottom left) and detector with beam stop (bottom right). 


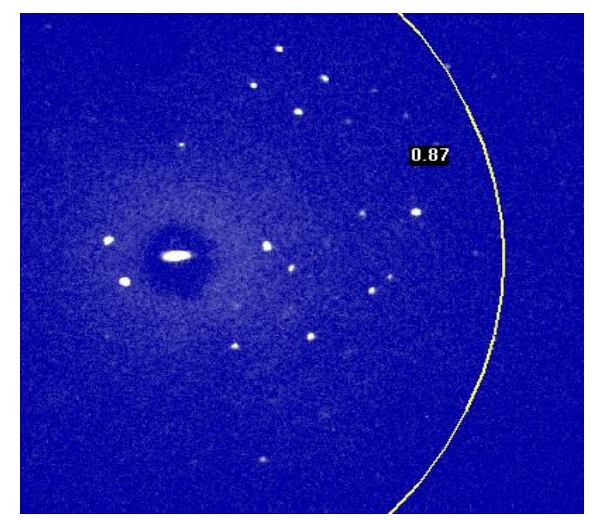

Figure 2: An example of the diffraction pattern obtained for compound 2, sample 2, as discussed in Section 4. The direct beam is the large elliptical white area to the left-hand side of the image.

Samples are placed on pre-aligned Mitegen ${ }^{\mathrm{TM}}$ micromounts fitted on non-magnetic SPINEtype pins $(0.64 \mathrm{~mm})$ to a magnetic base. Samples are glued in place with UV curable glue. Once covered with a plastic top, this is placed in the loading port and transferred on to the goniometer via the sample loader. Note that the plastic top is removed and stored during the sample loading process. A CD is placed in the CD drive of the instrument and the Bruker Kiosk interface software (Bruker, 2009) allows information about the crystal size, habit and color, as well as expected molecular formula to be input in to the software.

From this point, the system operates in a fully automated fashion. An initial unit-cell is determined (Bruker, 2009), followed by data collection (Bruker, 2009), structure solution (Sheldrick, 2008) and refinement (Sheldrick, 2008) using the Autostructure ${ }^{\mathrm{TM}}$ software. This data, along with the complete dataset and CIF file (Hall et al. 2006) are written to the CD for publication or further work, as required. The data is also presented in various formats, including html with JMol for viewing the chemical structure. Note that manual refinement is required for non-routine samples which exhibit issues such as twinning or disorder. Both the sample, with plastic top, and CD are ejected at the end of the experiment.

Initially, the instrument collects three runs of twelve frames each for the unit-cell determination at 10 seconds exposure per frame. The system aborts if insufficient diffraction is observed after the first run. In cases where diffraction is seen but the unit-cell cannot be determined, the software calculates how many additional frames have to be collected until an appropriate number of reflections are available for cell refinement. If a unit-cell is determined, data collection proceeds at exposure times between 5 and 60 seconds per frame, depending on the quality of the data obtained for the unit-cell. Thus, the minimum crystal size that can be analysed is dependent on the successful determination of the unit-cell at exposure times of 10 seconds per frame.

For samples where diffraction is observed but the unit-cell cannot be determined, the instrument collects the most data possible, i.e. a full sphere of data, by undertaking three $\omega$ scans of $180^{\circ}$. This is available for manual processing off-line and is seen for twinned crystals, for example, where the system knows it cannot use the indexing solution to solve the 
structure. If the unit-cell does not give a clear-cut indication of the likely symmetry of the crystal, a dataset corresponding to the lower symmetry is collected.

After the first $\omega$ scan of $180^{\circ}$ the software performs a Laue check and then re-refines the unit cell in the correct crystal system. It is at this point that the unit-cell displayed on the screen can change during data collection, since more information is now available compared to that used to determine the initial unit-cell initially. The information from the Laue check is used to determine the data collection strategy for the last two runs, for example changing the size of the $\omega$ scans.

The data collection time is chosen to acquire data with $\mathrm{I} / \sigma(\mathrm{I})$ of 1.2 at the $0.84 \AA$ limit. This is reflected in the resolution limit exhibited on screen during data collection, which can be lower for crystals which diffract poorly. In such cases, only the initial integration of the first run is done at $0.84 \AA$. The subsequent full integration of all runs is performed at the resolution determined from the first integration. After data collection, structure solution and refinement are undertaken using the entire dataset.

For experiments requiring manual off-line processing, this can be performed using the full functionality of the APEX2 software suite, which is supplied with the SMART X2S.

All experiments are stored on the internal hard drive, with the date and time noted, as well as written to $\mathrm{CD}$, which allows the administrator to monitor usage, for charging purposes etc. There is also a new option, which has just been announced in March 2010, which enables multiple users to pre-schedule the SMART X2S instrument time. 


\section{General Procedures}

All solvents used were HPLC grade or were distilled prior to use by the following methods: methylene chloride $\left(\mathrm{CH}_{2} \mathrm{Cl}_{2}\right)$ was distilled from phosphorous pentoxide, ethyl acetate was distilled from potassium carbonate, hexane was distilled prior to use. Organic phases were dried using anhydrous magnesium sulfate $\left(\mathrm{MgSO}_{4}\right)$. All commercial reagents were supplied by Sigma-Aldrich. All commercial reagents were used without further purification.

${ }^{1} \mathrm{H}(300 \mathrm{MHz})$ and ${ }^{13} \mathrm{C}(75.5 \mathrm{MHz}) \mathrm{NMR}$ spectra were recorded on a Brüker AVANCE 300 NMR spectrometer. ${ }^{1} \mathrm{H}(400 \mathrm{MHz})$ and ${ }^{13} \mathrm{C}(100.0 \mathrm{MHz}) \mathrm{NMR}$ spectra were recorded on a Brüker AVANCE 400 NMR spectrometer. All spectra were recorded at $20{ }^{\circ} \mathrm{C}$ in deuterated chloroform $\left(\mathrm{CDCl}_{3}\right)$ or deuterated dimethyl sulfoxide $\left(\mathrm{d}_{6}\right.$-DMSO). Chemical shifts $\left(\delta_{\mathrm{H}}\right.$ and $\left.\delta_{\mathrm{C}}\right)$ are reported in parts per million (ppm) relative to the TMS signal and coupling constants are expressed in Hertz (Hz). Splitting patterns for ${ }^{1} \mathrm{H}$ NMR and ${ }^{13} \mathrm{C}$ NMR spectra are designated as follows: singlet (s), broad singlet (bs), doublet (d), doublet of doublets (dd), doublet of doublets of doublets (ddd), triplet (t), doublet of triplets (dt), quartet (q), AB quartet $(\mathrm{ABq})$, multiplet $(\mathrm{m})$.

Infra-red spectra were recorded as potassium bromide (KBr) discs or as thin films on $\mathrm{NaCl}$ plates on a Perkin Elemer Paragon 1000 FT-IR spectrometer in the range $4,000-600 \mathrm{~cm}^{-1}$. Melting Point were measured on an Electrothermal 9100-melting point apparatus.

Microanalysis was performed by the Microanalysis Laboratory, UCC, Cork on Perkin-Elmer 240 and Exeter Analytical CE440 elemental analysers. Mass spectrometry was carried out using a Waters/Micromass Quattro Micro triplet quadrupole spectrometer (ESI) or a Kratosprofile HV-4 double focussing high resolution mass spectrometer (EI).

\section{Synthesis of precursor to 3}

A mixture of iso-butylamine (1.00 g, $1.36 \mathrm{~mL}, 13.65 \mathrm{mmol}), 2$-benzoylpyridine $(0.50 \mathrm{~g}, 2.73$ mmol), titanium(IV) ethoxide $(2.86 \mathrm{~mL}, 3.11 \mathrm{~g}, 13.65 \mathrm{mmol})$ and $10 \mathrm{~mL}$ of $\mathrm{CH}_{2} \mathrm{Cl}_{2}$ was heated at reflux overnight while stirring. The reaction was cooled to room temperature and deionised water $(5 \mathrm{~mL})$ was added slowly. The solution was filtered through celite to remove traces of solid material, and washed with $\mathrm{CH}_{2} \mathrm{Cl}_{2}(3 \times 5 \mathrm{~mL})$. The organic layer was separated and the aqueous layer was extracted with $\mathrm{CH}_{2} \mathrm{Cl}_{2}(3 \times 5 \mathrm{~mL})$. The combined organic layers were washed with brine $(5 \mathrm{~mL})$, dried $\left(\mathrm{MgSO}_{4}\right)$, filtered and concentrated to yield a colourless oil $(0.53 \mathrm{~g}, 81 \%) .{ }^{1} \mathrm{H}$ and ${ }^{13} \mathrm{C}$ NMR analysis showed that the reaction had gone to completion and the pure imine was obtained as $\mathrm{E} / \mathrm{Z}$ isomers in a ratio of 1.0:1.3; $v_{\max }$ $(\mathrm{NaCl}) / \mathrm{cm}^{-1} 1632(\mathrm{C}=\mathrm{N}), 1583\left(\mathrm{C}=\mathrm{N}\right.$, pyridine ring); $\delta_{\mathrm{H}}\left(300 \mathrm{MHz} ; \mathrm{CDCl}_{3}\right)$ Major isomer: $0.94\left(6 \mathrm{H}, \mathrm{d},{ }^{3} \mathrm{~J}_{\mathrm{H}-\mathrm{H}} 6.6, \mathrm{C}_{13} \mathrm{H}_{3}+\mathrm{C}_{14} H_{3}\right), 1.98-2.16\left(1 \mathrm{H}, \mathrm{m}, \mathrm{C}_{12} H\right), 3.28\left(2 \mathrm{H}, \mathrm{d},{ }^{3} \mathrm{~J}_{\mathrm{H}-\mathrm{H}} 6.6\right.$, $\left.\mathrm{C}_{11} \mathrm{H}_{2}\right), 7.18-8.04(8 \mathrm{H}, \mathrm{m}, \operatorname{Ar} H), 8.56\left(1 \mathrm{H}, \mathrm{d}\right.$ of d, $\left.{ }^{3} \mathrm{~J}_{\mathrm{HH}} 4.8,{ }^{4} \mathrm{~J}_{\mathrm{HH}} 1.5, \operatorname{ArC}_{1} \mathrm{H}\right)$, Minor isomer: $0.93\left(6 \mathrm{H}, \mathrm{d},{ }^{3} \mathrm{~J}_{\mathrm{H}-\mathrm{H}} 6.6, \mathrm{C}_{13} \mathrm{H}_{3}+\mathrm{C}_{14} H_{3}\right), 1.98-2.16\left(1 \mathrm{H}, \mathrm{m}, \mathrm{C}_{12} H\right), 3.19\left(2 \mathrm{H}, \mathrm{d},{ }^{3} \mathrm{~J}_{\mathrm{H}-\mathrm{H}}\right.$ 6.9, $\left.\mathrm{C}_{11} H_{2}\right), 7.15-8.07(8 \mathrm{H}, \mathrm{m}, \mathrm{ArH}), 8.75\left(1 \mathrm{H}, \mathrm{d}\right.$ of d, $\left.{ }^{3} \mathrm{~J}_{\mathrm{HH}} 4.8,{ }^{4} \mathrm{~J}_{\mathrm{HH}} 1.8, \operatorname{ArC}_{1} \mathrm{H}\right), \delta_{\mathrm{C}}(75$ $\left.\mathrm{MHz}, \mathrm{CDCl}_{3}\right)$ Major isomer: $20.9\left(C_{13}+C_{14}\right), 30.1\left(C_{12}\right), 61.8\left(C_{11}\right), 122.4\left(\mathrm{ArC}_{4}\right), 123.8$ $\left(\mathrm{ArC} C_{2}\right), 128.0\left(\mathrm{ArC} C_{9}\right), 128.2\left(\mathrm{ArC} C_{8}\right), 128.3\left(\mathrm{ArC} C_{10}\right), 136.2\left(\mathrm{ArC}_{3} \mathrm{H}\right), 136.5\left(\mathrm{Ar} C_{7}\right), 149.0$ $\left(\mathrm{Ar} C_{1} \mathrm{H}\right), 157.7\left(\mathrm{ArC}_{5}\right), 167.9(C=\mathrm{N})$, Minor isomer: $20.8\left(C_{13}+C_{14}\right), 30.2\left(C_{12}\right), 61.4\left(C_{11}\right)$, 
$123.0\left(\mathrm{ArC}_{2} / \mathrm{ArC}_{4}\right), 123.4\left(\mathrm{ArC} C_{2} / \mathrm{ArC}_{4}\right), 128.1\left(\mathrm{ArC} C_{8}+\mathrm{ArC} C_{9}\right), 129.8\left(\mathrm{ArC}_{10}\right), 136.1\left(\mathrm{ArC} C_{3} \mathrm{H}\right)$, $139.1\left(\mathrm{ArC}_{7}\right), 150.0\left(\mathrm{ArC} C_{1} \mathrm{H}\right), 156.1\left(\mathrm{ArC}_{5}\right), 165.8(C=\mathrm{N}) ; \mathrm{m} / z(\mathrm{ESI}) 239(\mathrm{M}+1,100 \%), 240$ $(\mathrm{M}+2,39 \%)$; HRMS calcd. for $\mathrm{C}_{16} \mathrm{H}_{18} \mathrm{~N}_{2} 239.1548$, found 239.1541 .

\section{Synthesis of 3}

A mixture of palladium(II) chloride-1,5-cyclooctadiene $(0.10 \mathrm{~g}, 0.35 \mathrm{mmol})$, iso-butyl(phenyl-pyridin-2-yl-methylene)-amine $(0.09 \mathrm{~g}, 0.38 \mathrm{mmol})$ and $10 \mathrm{~mL}$ of $\mathrm{CH}_{2} \mathrm{Cl}_{2}$ was stoppered and stirred for $6 \mathrm{~h}$. No precipitate formed so the reaction mixture was heated at reflux overnight. The product was concentrated to a bright orange solid under reduced pressure. It was then dissolved in the minimum amount of $\mathrm{CH}_{2} \mathrm{Cl}_{2}$ and layered with hexane. It was left overnight and the solid precipitated out. This was collected and washed with hexane $(3 \times 5 \mathrm{~mL})$ to give the desired compound as bright orange crystals $(0.12 \mathrm{~g}, 86 \%) ; v_{\max }$ $(\mathrm{KBr}) / \mathrm{cm}^{-1} 1586(\mathrm{C}=\mathrm{N}) ; \delta_{\mathrm{H}}\left(400 \mathrm{MHz} ; \mathrm{CDCl}_{3}\right) 0.80\left(6 \mathrm{H}, \mathrm{d},{ }^{3} \mathrm{~J}_{\mathrm{H}-\mathrm{H}} 6.8, \mathrm{C}_{13} \mathrm{H}_{3}+\mathrm{C}_{14} \mathrm{H}_{3}\right), 2.71$ $\left(1 \mathrm{H}\right.$, septet, $\left.{ }^{3} \mathrm{~J}_{\mathrm{H}-\mathrm{H}} 6.8, \mathrm{C}_{12} H\right), 3.66\left(2 \mathrm{H}, \mathrm{d},{ }^{3} \mathrm{~J}_{\mathrm{H}-\mathrm{H}} 6.4, \mathrm{C}_{11} H_{2}\right), 7.12\left(1 \mathrm{H}, \mathrm{d}\right.$ of d, ${ }^{3} \mathrm{~J}_{\mathrm{HH}} 8.0,{ }^{4} \mathrm{~J}_{\mathrm{HH}}$ 0.8, $\left.\mathrm{ArC}_{10} \mathrm{H}\right), 7.34$ - $7.36(2 \mathrm{H}, \mathrm{m}, \mathrm{ArH}), 7.59$ - $7.67(4 \mathrm{H}, \mathrm{m}, \mathrm{ArH}), 7.98\left(1 \mathrm{H}, \mathrm{t}\right.$ of d, ${ }^{3} \mathrm{~J}_{\mathrm{HH}} 8.0$, $\left.{ }^{4} \mathrm{~J}_{\mathrm{HH}} 1.6, \mathrm{ArC}_{4} \mathrm{H}\right), 9.46\left(1 \mathrm{H}, \mathrm{d}\right.$ of d$\left.,{ }^{3} \mathrm{~J}_{\mathrm{HH}} 5.6,{ }^{4} \mathrm{~J}_{\mathrm{HH}} 1.2, \mathrm{ArC}_{1} \mathrm{H}\right)$. Found $\mathrm{C}, 46.09 ; \mathrm{H}, 4.31 ; \mathrm{N}$, 6.67; $\mathrm{Cl}, 16.87 ; \mathrm{C}_{16} \mathrm{H}_{18} \mathrm{~N}_{2} \mathrm{Cl}_{2} \mathrm{Pd}$ requires C, 46.23; H, 4.36; N, 6.74; Cl, 17.06.

\section{Synthesis of precursor to 4}

A mixture of $n$-pentylamine $(1.19 \mathrm{~g}, 1.58 \mathrm{~mL}, 13.65 \mathrm{mmol}), 2$-benzoylpyridine $(0.50 \mathrm{~g}, 2.73$ $\mathrm{mmol})$, titanium(IV) ethoxide $(2.86 \mathrm{~mL}, 3.11 \mathrm{~g}, 13.65 \mathrm{mmol})$ and $10 \mathrm{~mL}$ of dichloromethane was refluxed overnight with stirring. The reaction was cooled to room temperature and deionised water $(5 \mathrm{~mL})$ was added slowly. The solution was filtered through celite to remove traces of solid material, and washed with $\mathrm{CH}_{2} \mathrm{Cl}_{2}(3 \times 5 \mathrm{~mL})$. The organic layer was separated and the aqueous layer was extracted with $\mathrm{CH}_{2} \mathrm{Cl}_{2}(3 \times 5 \mathrm{~mL})$. The combined organic layers were washed with brine $(5 \mathrm{~mL})$, dried $\left(\mathrm{MgSO}_{4}\right)$, filtered and concentrated to yield a colourless oil $(0.57 \mathrm{~g}, 83 \%) .{ }^{1} \mathrm{H}$ and ${ }^{13} \mathrm{C}$ NMR analysis showed that the reaction had gone to completion and the pure imine was obtained as $\mathrm{E} / \mathrm{Z}$ isomers in a ratio of 1.0:1.4; $v_{\max }$ $(\mathrm{NaCl}) / \mathrm{cm}^{-1} 1629(\mathrm{C}=\mathrm{N}), 1583(\mathrm{C}=\mathrm{N}$, pyridine ring $) ; \delta_{\mathrm{H}}\left(300 \mathrm{MHz} ; \mathrm{CDCl}_{3}\right)$ Major isomer: $0.85-0.89\left(3 \mathrm{H}, \mathrm{m}, \mathrm{C}_{15} \mathrm{H}_{3}\right), 1.20-1.36\left(4 \mathrm{H}, \mathrm{m}, \mathrm{C}_{13} \mathrm{H}_{2}+\mathrm{C}_{14} \mathrm{H}_{2}\right), 1.66-1.77\left(2 \mathrm{H}, \mathrm{m}, \mathrm{C}_{12} \mathrm{H}_{2}\right)$, $3.45\left(2 \mathrm{H}, \mathrm{t},{ }^{3} \mathrm{~J}_{\mathrm{H}-\mathrm{H}} 7.2, \mathrm{C}_{11} H_{2}\right), 7.19-7.93(8 \mathrm{H}, \mathrm{m}, \mathrm{ArH}), 8.58\left(1 \mathrm{H}, \mathrm{d}\right.$ of d, ${ }^{3} \mathrm{~J}_{\mathrm{HH}} 4.8,{ }^{4} \mathrm{~J}_{\mathrm{HH}} 1.5$, $\left.\mathrm{ArC}_{1} \mathrm{H}\right)$, Minor isomer: $0.85-0.89\left(3 \mathrm{H}, \mathrm{m}, \mathrm{C}_{15} \mathrm{H}_{3}\right), 1.20-1.36\left(4 \mathrm{H}, \mathrm{m}, \mathrm{C}_{13} \mathrm{H}_{3}+\mathrm{C}_{14} \mathrm{H}_{3}\right), 1.66$ $1.77\left(2 \mathrm{H}, \mathrm{m}, \mathrm{C}_{12} \mathrm{H}_{2}\right), 3.36\left(2 \mathrm{H}, \mathrm{t},{ }^{3} \mathrm{~J}_{\mathrm{H}-\mathrm{H}} 7.2, \mathrm{C}_{11} \mathrm{H}_{2}\right), 7.16-7.96(8 \mathrm{H}, \mathrm{m}, \mathrm{ArH}), 8.75(1 \mathrm{H}, \mathrm{d}$ of $\left.\mathrm{d},{ }^{3} \mathrm{~J}_{\mathrm{HH}} 4.8,{ }^{4} \mathrm{~J}_{\mathrm{HH}} 1.5, \operatorname{ArC}_{1} \mathrm{H}\right), \delta_{\mathrm{C}}\left(75 \mathrm{MHz}, \mathrm{CDCl}_{3}\right)$ Major isomer: $14.0\left(C_{15}\right), 22.5\left(C_{14}\right)$, $29.8\left(C_{13}\right), 30.8\left(C_{12}\right), 54.2\left(C_{11}\right), 122.6\left(\mathrm{Ar} C_{4}\right), 123.8\left(\mathrm{ArC}_{2}\right), 127.9\left(\operatorname{Ar} C_{9}\right), 128.2\left(\operatorname{Ar} C_{8}\right)$, $128.3\left(\mathrm{ArC}_{10}\right), 136.2\left(\mathrm{ArC}_{3} \mathrm{H}\right), 136.4\left(\mathrm{ArC}_{7}\right), 149.1\left(\mathrm{ArC} C_{1} \mathrm{H}\right), 157.6\left(\mathrm{ArC}_{5}\right), 167.9(C=\mathrm{N})$, Minor isomer: $14.0\left(C_{15}\right), 22.5\left(C_{14}\right), 29.7\left(C_{13}\right), 30.8\left(C_{12}\right), 53.7\left(C_{11}\right), 123.0\left(\mathrm{Ar} C_{4}\right), 123.3$ $\left(\mathrm{Ar} C_{2}\right), 128.0\left(\mathrm{ArC}_{8} / \mathrm{Ar} C_{9}\right), 128.1\left(\mathrm{ArC}_{8} / \mathrm{ArC} C_{9}\right), 129.9\left(\mathrm{ArC} C_{10}\right), 136.1\left(\mathrm{ArC} C_{3} \mathrm{H}\right), 139.0\left(\mathrm{ArC} C_{7}\right)$, $150.0\left(\mathrm{Ar} C_{1} \mathrm{H}\right), 156.1\left(\mathrm{Ar} C_{5}\right), 165.9(C=\mathrm{N}) ; \mathrm{m} / \mathrm{z}(\mathrm{ESI}) 253(\mathrm{M}+1,100 \%), 254(\mathrm{M}+2,31 \%)$, $255(\mathrm{M}+3,3 \%)$; HRMS calcd. for $\mathrm{C}_{17} \mathrm{H}_{20} \mathrm{~N}_{2} 253.1705$, found 253.1712 . 


\section{Synthesis of 4}

A mixture of palladium(II) chloride-1,5-cyclooctadiene ${ }^{1}(0.10 \mathrm{~g}, 0.35 \mathrm{mmol})$, pentyl-(phenylpyridin-2-yl-methylene)-amine $(0.09 \mathrm{~g}, 0.36 \mathrm{mmol})$ and $10 \mathrm{~mL}$ of dichloromethane was stoppered and stirred for $6 \mathrm{~h}$. No precipitate formed so the reaction mixture was heated at reflux overnight. The product was concentrated to a bright orange solid under reduced pressure. It was then dissolved in the minimum amount of $\mathrm{CH}_{2} \mathrm{Cl}_{2}$ and layered with hexane. It was left overnight and the solid precipitated out. This was collected and washed with hexane $(3 \times 5 \mathrm{~mL})$ to give the desired compound as a bright orange crystalline solid $(0.11 \mathrm{~g}, 79 \%)$; $v_{\max }(\mathrm{KBr}) / \mathrm{cm}^{-1} 1588(\mathrm{C}=\mathrm{N}) ; \delta_{\mathrm{H}}\left(400 \mathrm{MHz} ; \mathrm{CDCl}_{3}\right) 0.78\left(3 \mathrm{H}, \mathrm{t},{ }^{3} \mathrm{~J}_{\mathrm{H}-\mathrm{H}} 6.8 \mathrm{C}_{15} H_{3}\right), 1.12-1.18$ $\left(4 \mathrm{H}, \mathrm{m}, \mathrm{C}_{13} \mathrm{H}_{2}+\mathrm{C}_{14} \mathrm{H}_{2}\right), 1.77-1.84\left(2 \mathrm{H}, \mathrm{m}, \mathrm{C}_{12} \mathrm{H}_{2}\right), 3.73\left(2 \mathrm{H}, \mathrm{t},{ }^{3} \mathrm{~J}_{\mathrm{H}-\mathrm{H}} 7.6, \mathrm{C}_{11} H_{2}\right), 7.07(1 \mathrm{H}, \mathrm{d}$ of $\mathrm{d},{ }^{3} \mathrm{~J}_{\mathrm{HH}} 8.0,{ }^{4} \mathrm{~J}_{\mathrm{HH}}$ 0.8, $\left.\operatorname{ArC}_{10} \mathrm{H}\right), 7.43-7.45(2 \mathrm{H}, \mathrm{m}, \operatorname{ArH}), 7.59-7.65(4 \mathrm{H}, \mathrm{m}, \operatorname{ArH}), 7.95$ $\left(1 \mathrm{H}, \mathrm{t}\right.$ of d, $\left.{ }^{3} \mathrm{~J}_{\mathrm{HH}} 7.6,{ }^{4} \mathrm{~J}_{\mathrm{HH}} 1.6, \mathrm{ArC}_{4} \mathrm{H}\right), 9.39\left(1 \mathrm{H}, \mathrm{d}\right.$ of d, $\left.{ }^{3} \mathrm{~J}_{\mathrm{HH}} 5.6,{ }^{4} \mathrm{~J}_{\mathrm{HH}} 1.2, \mathrm{ArC}_{1} \mathrm{H}\right)$. Found C, 47.37; H, 4.49; N, 6.42; Cl, 16.20; $\mathrm{C}_{17} \mathrm{H}_{20} \mathrm{~N}_{2} \mathrm{Cl}_{2} \mathrm{Pd}$ requires $\mathrm{C}, 47.52 ; \mathrm{H}, 4.69 ; \mathrm{N}, 6.52 ; \mathrm{Cl}$, 16.50 .

\section{Synthesis of 11}

4-Nitrobenzene thiol $(1.71 \mathrm{~g}, 11.0 \mathrm{mmol})$ was added dropwise to a stirred suspension of $\mathrm{NaH}$ $(0.46 \mathrm{~g}, 60 \%$ dispersion in oil, $11.6 \mathrm{mmol})$ in $\mathrm{DMF}(15 \mathrm{~mL})$ under $\mathrm{N}_{2}$ at $0{ }^{\circ} \mathrm{C}$. The reaction was stirred for 20 minutes and 4-iodobenzyl bromide $(3.27 \mathrm{~g}, 11.0 \mathrm{mmol})$ in DMF (1 mL) was then slowly added over 20 minutes. The reaction mixture was stirred for a further $16 \mathrm{~h}$ at room temperature. Water $(50 \mathrm{~mL})$ and $\mathrm{CH}_{2} \mathrm{Cl}_{2}(30 \mathrm{~mL})$ were added and the phases separated. The organic layer was concentrated under reduced pressure. The product was dissolved in $\mathrm{CH}_{2} \mathrm{Cl}_{2}(30 \mathrm{~mL})$, washed with $2 \mathrm{M} \mathrm{HCl}(3 \times 20 \mathrm{~mL})$ and brine $(1 \times 20 \mathrm{~mL})$, dried over $\mathrm{MgSO}_{4}$ and concentrated under reduced pressure to give the crude product. Purification by recrystallisation from hot methanol afforded the title compound as a yellow solid $(3.62 \mathrm{~g}, 91$ \%), mp 138-140 ${ }^{\circ} \mathrm{C} ; v_{\max }(\mathrm{KBr}) / \mathrm{cm}^{-1} 1594,1578,1506$ (asymmetric $\mathrm{NO}_{2}$ stretch), 1331 (symmetric $\mathrm{NO}_{2}$ stretch), 475 (C-I stretch); $\delta_{\mathrm{H}}(300 \mathrm{MHz})\left(\mathrm{CDCl}_{3}\right) 4.18\left(2 \mathrm{H}, \mathrm{s}, \mathrm{CH}_{2}\right), 7.08$ $7.17(2 \mathrm{H}, \mathrm{m}, \mathrm{ArCH}), 7.28-7.36$ (2H, m, ArH), 7.62-7.70 (2H, m, ArH), 8.06-8.15 (2H, m, ArH); $\delta_{\mathrm{c}}(75.5 \mathrm{MHz})\left(\mathrm{CDCl}_{3}\right) 36.6\left(\mathrm{CH}_{2}, \mathbf{C H}_{2}\right), 93.3(\mathrm{C}$, aromatic $\mathbf{C}-\mathrm{I}), 124.0,126.9,130.6$ $(\mathrm{CH}, 3 \times$ aromatic $\mathbf{C H}), 135.3(\mathrm{C}$, aromatic $\mathrm{C}), 138.0(\mathrm{CH}$, aromatic $\mathrm{CH}), 146.5(\mathrm{C}$, aromatic

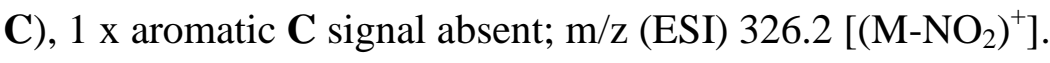

\section{Synthesis of 15}

To a solution of triethylamine $(2 \mathrm{~mL}, 1.45 \mathrm{~g}, 14.35 \mathrm{mmol})$ and sulfathiazole $(3.99 \mathrm{~g}, 15.64$ $\mathrm{mmol})$ in dry methanol $(26 \mathrm{~mL})$, was slowly added di-tert-butyldicarbonate $(4.12 \mathrm{~g}, 18.90$ mmol) in dry methanol $(1 \mathrm{~mL})$ at $0-5{ }^{\circ} \mathrm{C}$. The resulting suspension was stirred at room temperature for $24 \mathrm{~h}$. TLC analysis $\left(\mathrm{CH}_{2} \mathrm{Cl}_{2}\right.$ : methanol, 90:10) showed product and starting material and the solution was $\mathrm{pH}=8$, so additional di-tert-butyl dicarbonate $(1.35 \mathrm{~g}, 6.21$ $\mathrm{mmol})$ in methanol $(3 \mathrm{~mL})$ was added along with triethylamine $(0.9 \mathrm{~mL})$ and the reaction stirred at room temperature for $3 \mathrm{~h}$. The reaction mixture was separated between $\mathrm{HCl}(2 \mathrm{M}$, $30 \mathrm{~mL}$ ) and ethyl acetate $(30 \mathrm{~mL})$. The aqueous layer was separated and extracted with ethyl acetate $(3 \times 20 \mathrm{~mL})$, washed with brine $(10 \mathrm{~mL})$ and the combined organic extracts were 
dried and concentrated to give a crude yellow solid, which was immediately recrystallised twice from cold methanol/water to give a white solid (0.685 g, $1.93 \mathrm{mmol}, 12 \%)$, mp 188-190 ${ }^{\circ} \mathrm{C} . \delta_{\mathrm{H}}\left(300 \mathrm{MHz} ; \mathrm{DMSO}-\mathrm{d}_{6}\right) 1.48\left(9 \mathrm{H}, \mathrm{s}, \mathrm{C}\left(\mathrm{CH}_{3}\right)_{3}, 6.79-6.80\left(1 \mathrm{H}, \mathrm{d},{ }^{3} \mathrm{~J}=4.62 \mathrm{~Hz}, \mathrm{CHCH}\right)\right.$, 7.22-7.23 (1H, d, $\left.{ }^{3} \mathrm{~J}=4.65 \mathrm{~Hz}, \mathrm{CHCH}\right), 7.55-7.58\left(2 \mathrm{H}, \mathrm{d},{ }^{3} \mathrm{~J}=8.82 \mathrm{~Hz}, \operatorname{Ar}-H\right), 7.67-7.69(2 \mathrm{H}$, $\left.\mathrm{d},{ }^{3} \mathrm{~J}=8.79 \mathrm{~Hz}, \mathrm{Ar}-H\right), 9.72(1 \mathrm{H}$, br s, $\mathrm{N} H) ; \delta_{\mathrm{C}}(75 \mathrm{MHz}$; DMSO-d 6$) 26.88\left(-\mathrm{CH}_{3}\right), 78.58(\mathrm{C}-$ $\left.\mathrm{CH}_{3}\right), 106.84,124.44(-\mathrm{CH}), 116.29(2 \times \mathrm{Ar}-\mathrm{CH}), 125.77(2 \times \mathrm{Ar}-\mathrm{CH}), 134.09,141.72$ (Quaternary $\mathrm{Ar}-C$ ), $151.39(C=\mathrm{O}) ; \quad v_{\max }\left(\mathrm{KBr} / \mathrm{cm}^{-1}\right) 3345(\mathrm{~N}-\mathrm{H}), 3117$ (ArylC-H), 1718 $(\mathrm{C}=\mathrm{O}), 1593(\mathrm{C}=\mathrm{C}), 1330,1147\left(\mathrm{SO}_{2}\right)$; Found: $\mathrm{C}, 47.23 ; \mathrm{H}, 4.87 ; \mathrm{N}, 12.02 ; \mathrm{C}_{14} \mathrm{H}_{17} \mathrm{~N}_{3} \mathrm{O}_{4} \mathrm{~S}_{2}$ requires $\mathrm{C}, 47.31 ; \mathrm{H}, 4.82 ; \mathrm{N}, 11.82$.

\section{Crystallisation of 16}

Sulfaguanidine $(0.214 \mathrm{~g}, 1.000 \mathrm{mmol})$ was dissolved in bulk acetone $(40 \mathrm{~mL})$ and allowed to stand at ambient temperature over 2 days to afford white needle crystals $(0.196 \mathrm{~g}, 92 \%)$ suitable for single X-ray diffraction.

\section{Synthesis of 17}

Triphenylphosphine oxide $(0.278 \mathrm{~g}, 1.000 \mathrm{mmol})$ and thiobenzamide $(0.137 \mathrm{~g}, 1.000 \mathrm{mmol})$ were dissolved in acetone $(20 \mathrm{~mL})$. The solution was allowed to stand at ambient temperature over 3 days to afford a yellow solid $(0.336 \mathrm{~g}, 81 \%)$, mp $129-131{ }^{\circ} \mathrm{C}$. Found: $\mathrm{C}, 71.95 ; \mathrm{H}$, 3.58; N, 5.40; S, 8.20; $\mathrm{C}_{25} \mathrm{H}_{22}$ NOPS requires $\mathrm{C}, 72.27 ; \mathrm{H}, 3.37 ; \mathrm{N}, 5.34 ; \mathrm{S}, 7.72$. 

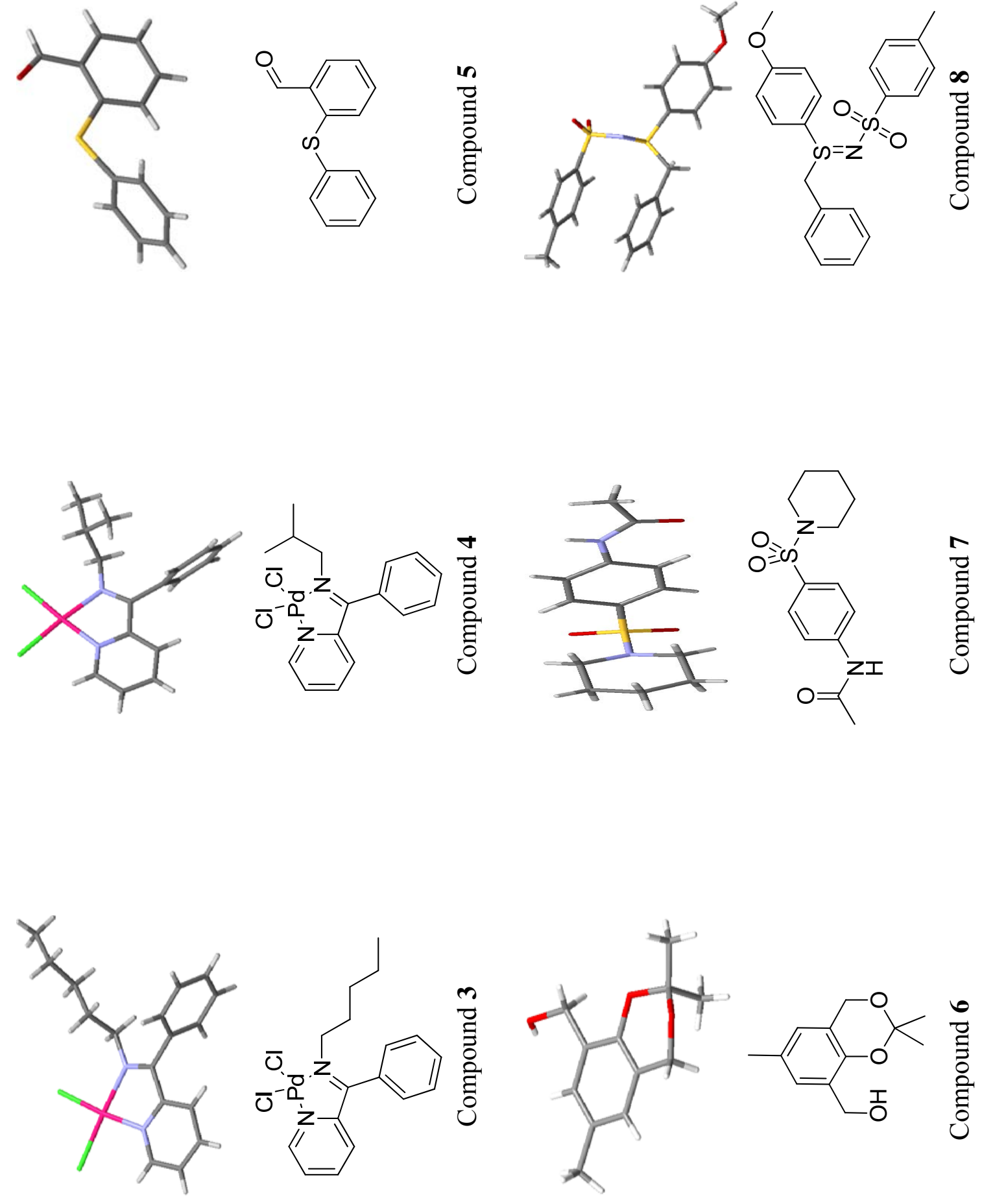


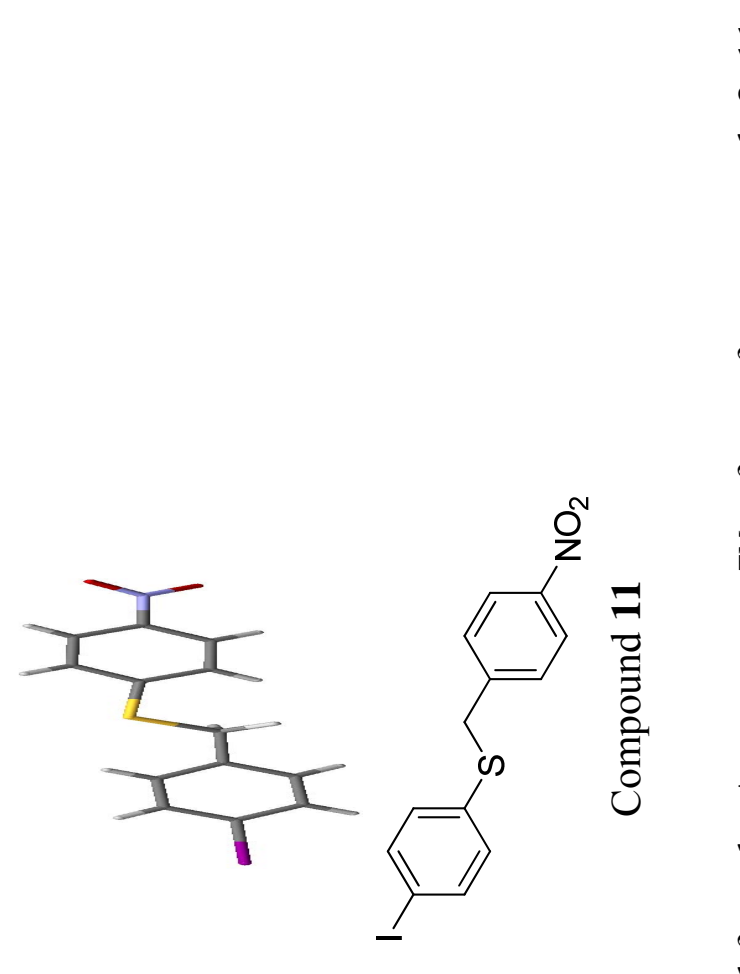

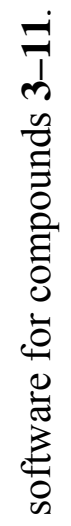

过

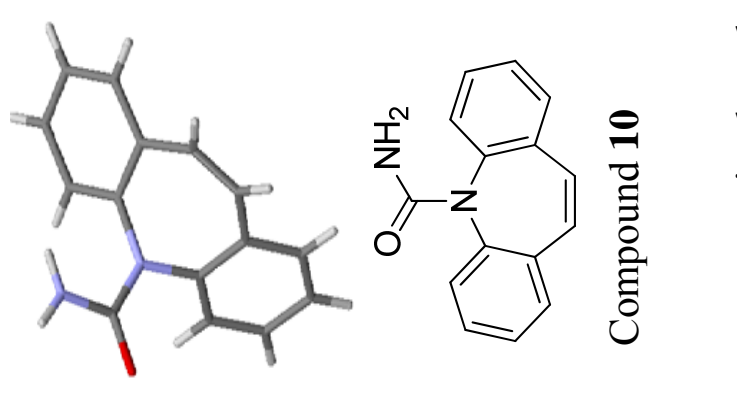

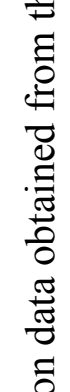
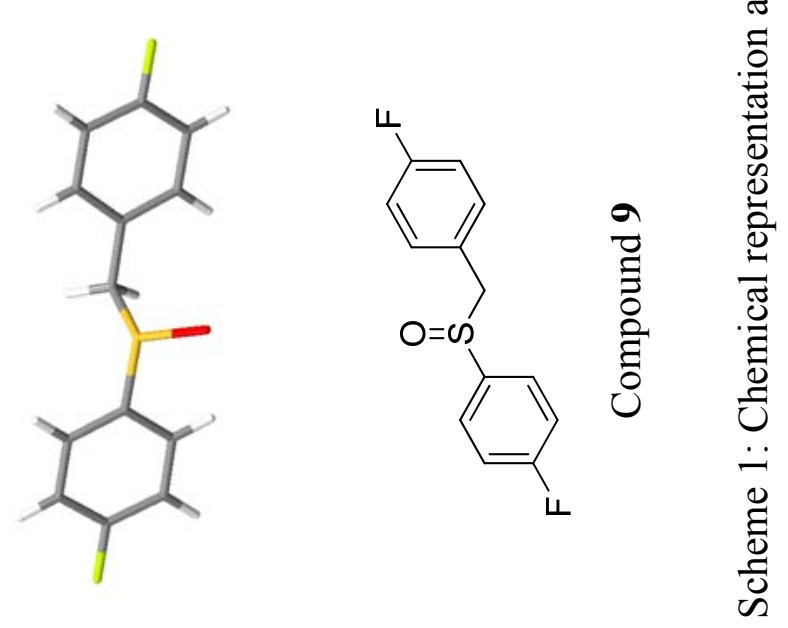


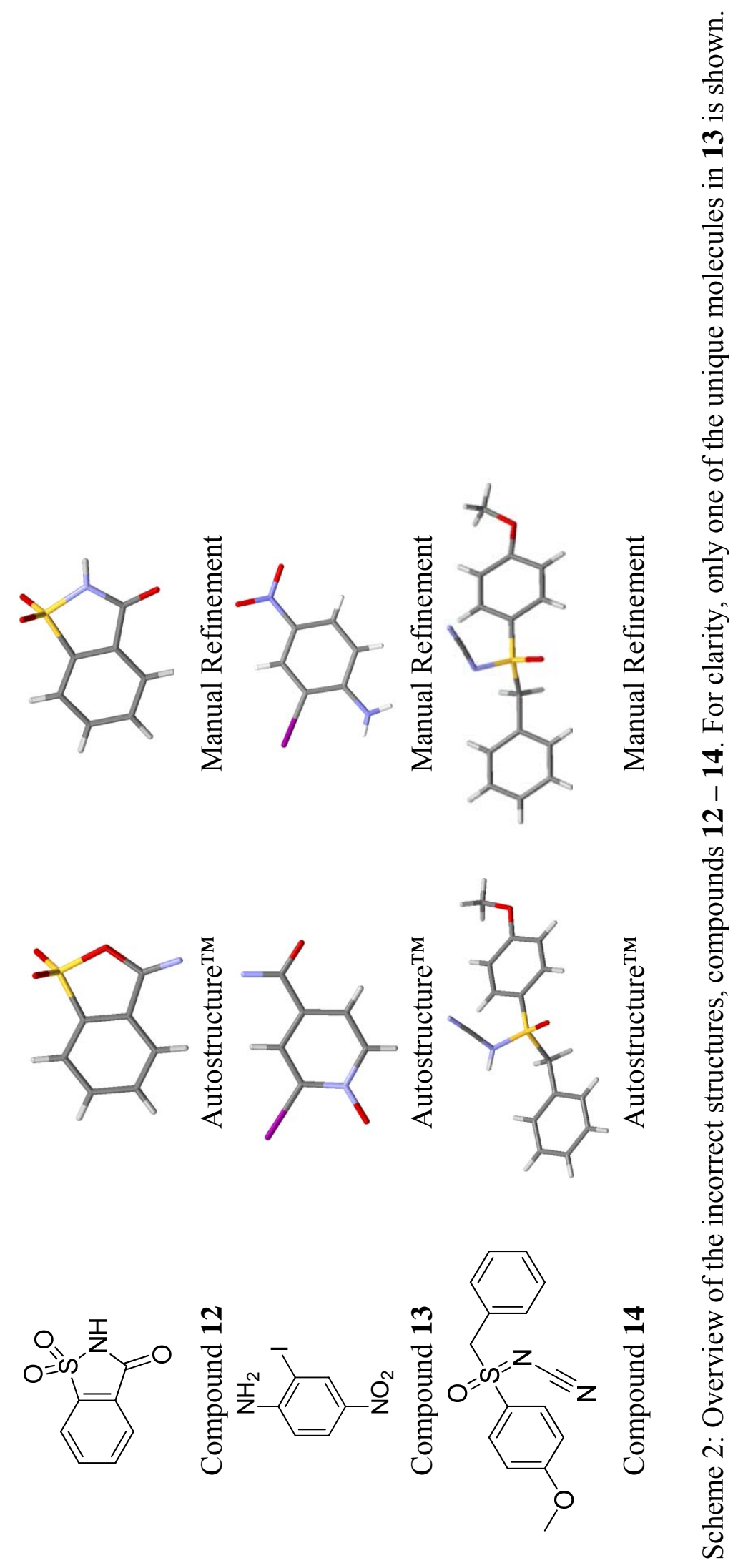



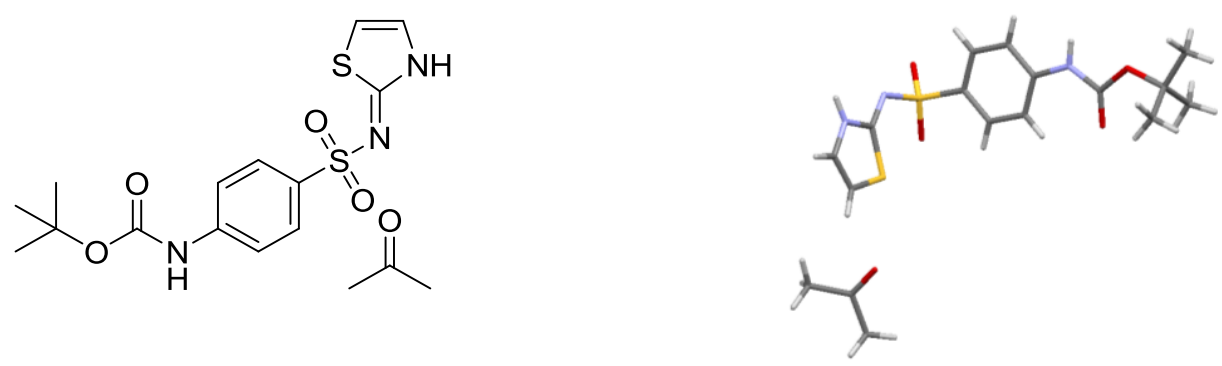

Compound 15
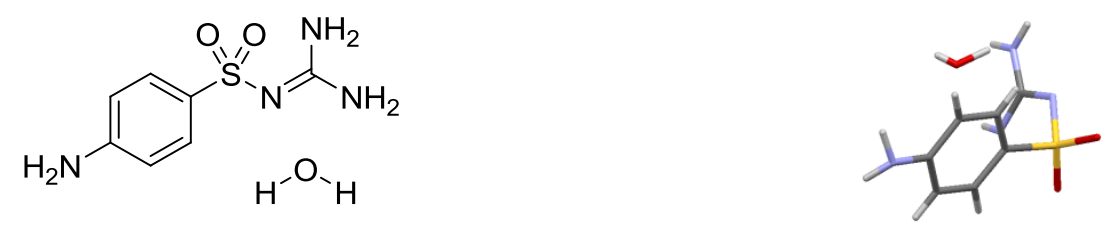

Compound 16
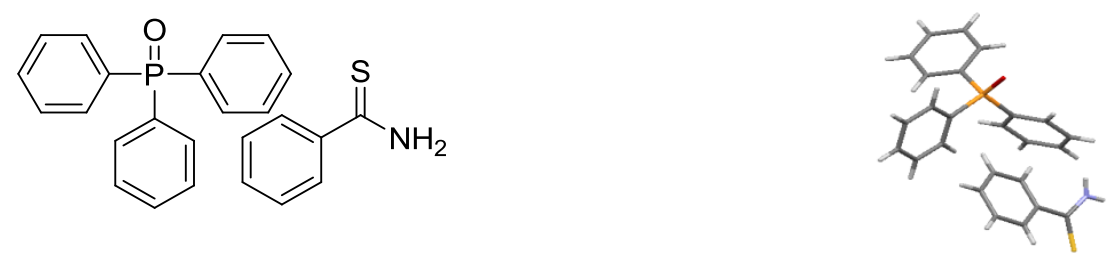

Compound 17

Scheme 3: Multi-component systems for compounds 15-17. 
Table 1: Crystallographic Data for Compound 1.

\begin{tabular}{|c|c|c|c|}
\hline Position of crystal & Centre & Below & Side \\
\hline $\begin{array}{l}\text { Exposure time per } \\
\text { frame, } \mathrm{s}\end{array}$ & 10 & 60 & 60 \\
\hline Resolution, $\AA$ & 0.84 & 0.92 & 0.84 \\
\hline $\begin{array}{c}\text { Unit-cell dimensions, } \\
\AA \&^{\circ}\end{array}$ & $\begin{array}{l}a=24.003(3) \\
b=4.7435(6) \\
c=10.8255(12) \\
\beta=90.185(4)\end{array}$ & $\begin{array}{l}a=4.736(3) \\
b=10.819(5) \\
c=12.206(6) \\
\alpha=89.727(15) \\
\beta=78.904(14) \\
\gamma=89.977(18)\end{array}$ & $\begin{array}{l}a=24.012(3) \\
b=4.7429(5) \\
c=10.8321(13) \\
\beta=90.197(4)\end{array}$ \\
\hline$V, \AA^{3}$ & $1232.6(3)$ & $613.6(5)$ & $1233.6(3)$ \\
\hline Total reflections & 3757 & 4509 & 3662 \\
\hline Unique reflections & 1094 & 1596 & 1097 \\
\hline $\begin{array}{l}\text { Observed reflections, } \\
\qquad I>2 \sigma(I)\end{array}$ & 894 & 628 & 960 \\
\hline$R_{\text {int }}$ & 0.031 & 0.200 & 0.024 \\
\hline GooF & 1.16 & 0.98 & 1.20 \\
\hline$R 1, I>2 \sigma(I)$ & 0.036 & 0.099 & 0.038 \\
\hline$w R 2$, all data & 0.132 & 0.321 & 0.122 \\
\hline$\Delta \rho_{\max }, \Delta \rho_{\min }$ & $0.24,-0.33$ & $0.42,-0.42$ & $0.24,-0.33$ \\
\hline $\begin{array}{c}\text { Mean C-C Bond } \\
\text { Precision, } \AA\end{array}$ & 0.0037 & 0.0197 & 0.0033 \\
\hline
\end{tabular}




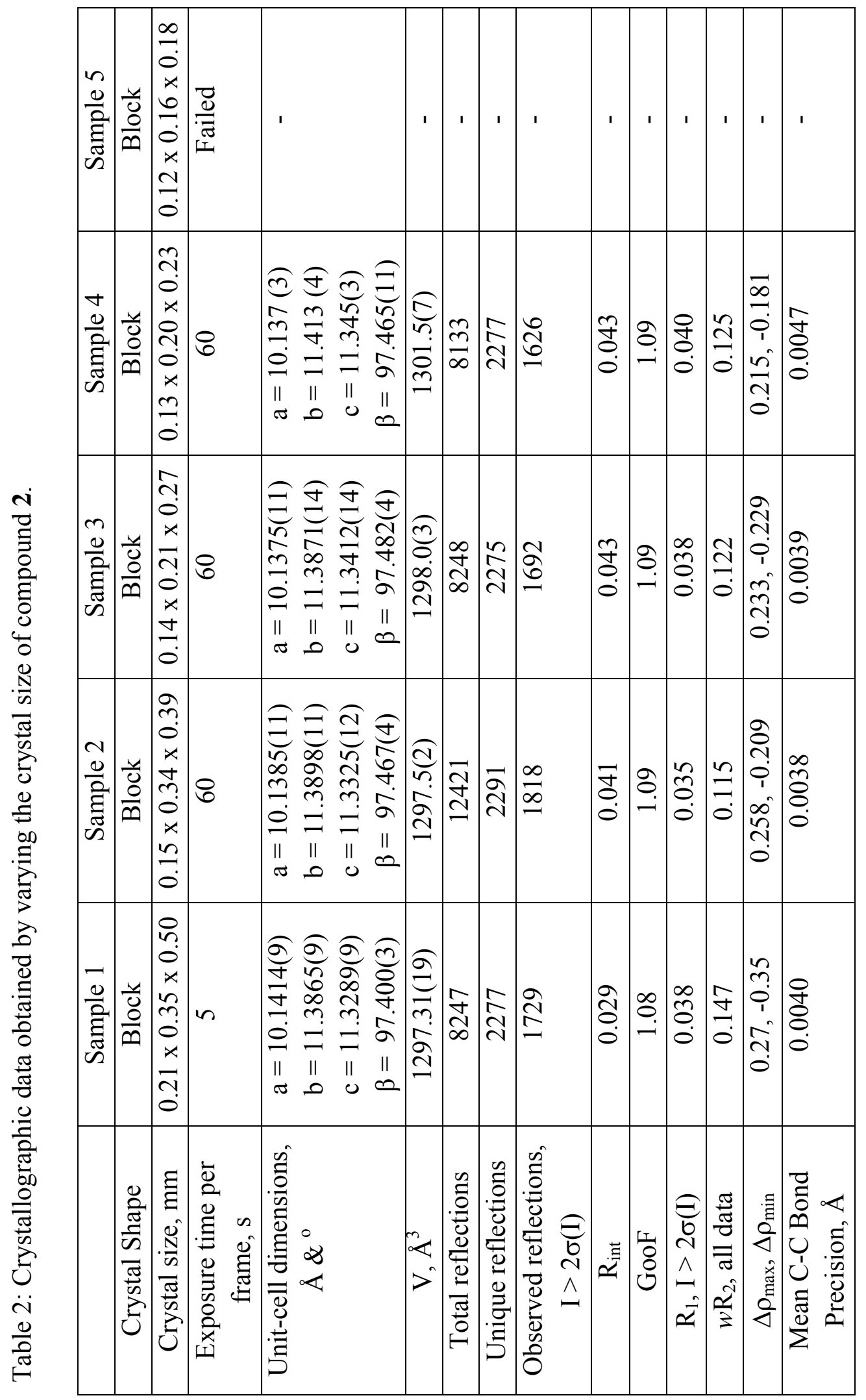




\begin{tabular}{|c|c|c|c|c|c|c|c|c|c|c|c|c|}
\hline 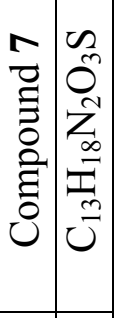 & 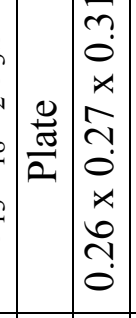 & & 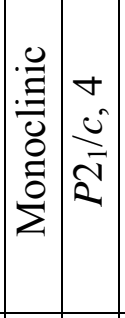 & 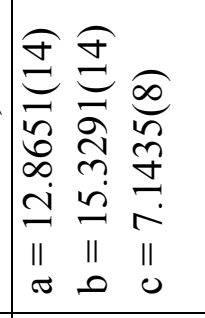 & 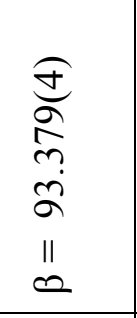 & 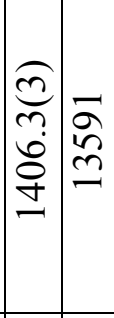 & $\left|\begin{array}{l}\tilde{N} \\
\tilde{N} \\
\mathcal{N}\end{array}\right|$ & 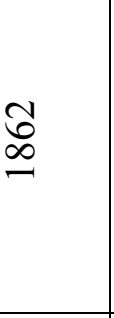 & $\begin{array}{l}\vec{t} \\
0 \\
0\end{array}$ & ơ & & \\
\hline 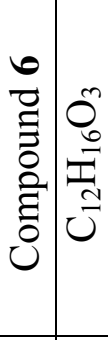 & 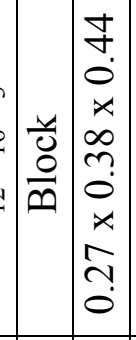 & & 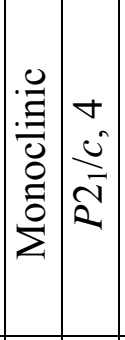 & 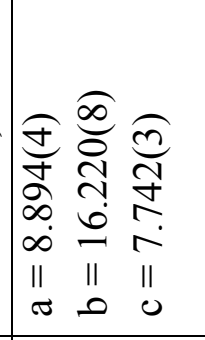 & 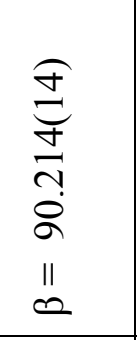 & 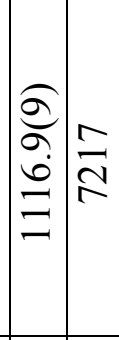 & & $\begin{array}{l}\stackrel{\infty}{\simeq} \\
\stackrel{\sim}{\simeq}\end{array}$ & 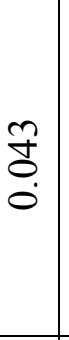 & 草 & & \\
\hline 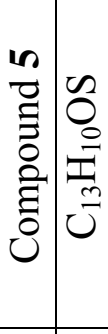 & 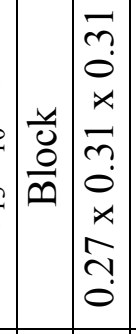 & & 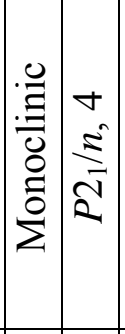 & 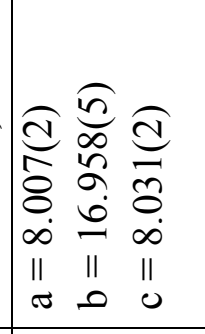 & $\begin{array}{l}\widehat{Q} \\
\stackrel{0}{a} \\
0 \\
\dot{a} \\
\| \\
11 \\
0\end{array}$ & 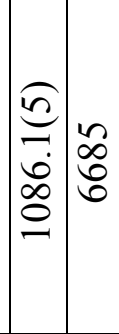 & $\frac{\circ}{9}$ & ర్ల & $\begin{array}{l}\vec{t} \\
0 \\
0\end{array}$ & & & \\
\hline 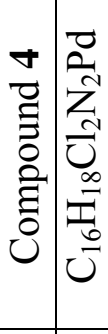 & 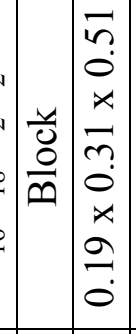 & & 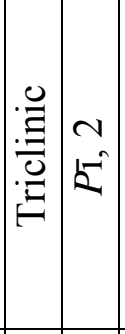 & 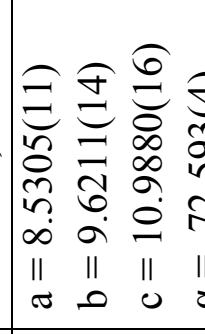 & 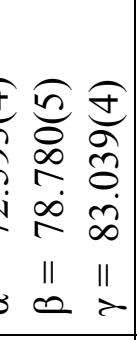 & & & 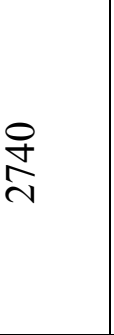 & त्र & & & $\begin{array}{l}\infty \\
0 \\
0 \\
0 \\
0 \\
0 \\
0\end{array}$ \\
\hline 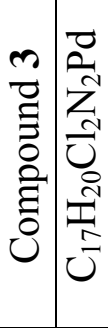 & 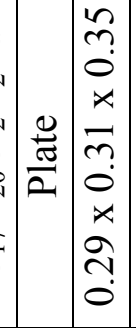 & & 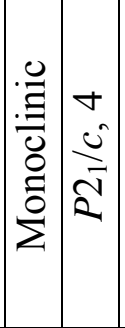 & 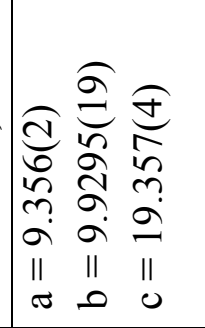 & 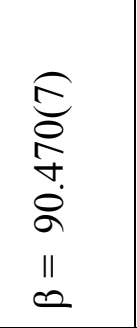 & 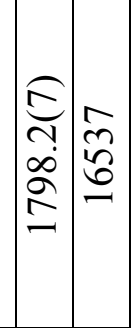 & $\approx$ & $\overrightarrow{\widetilde{d}}$ & 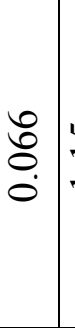 & & $\begin{array}{l}0 \\
\vec{T} \\
0 \\
0\end{array}$ & 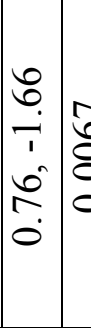 \\
\hline 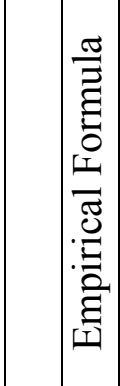 & 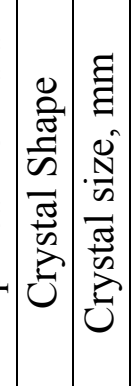 & 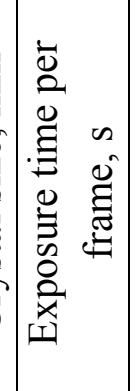 & 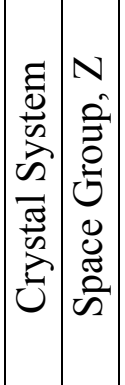 & 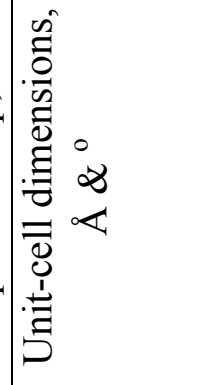 & & 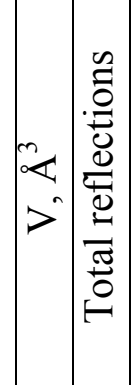 & $\left|\begin{array}{l}\frac{0}{\tilde{D}} \\
0 \\
0\end{array}\right|$ & 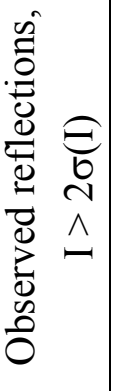 & & & & 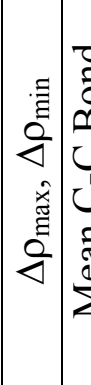 \\
\hline
\end{tabular}




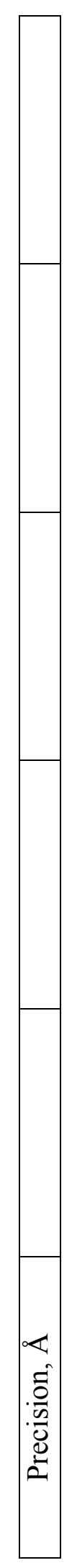

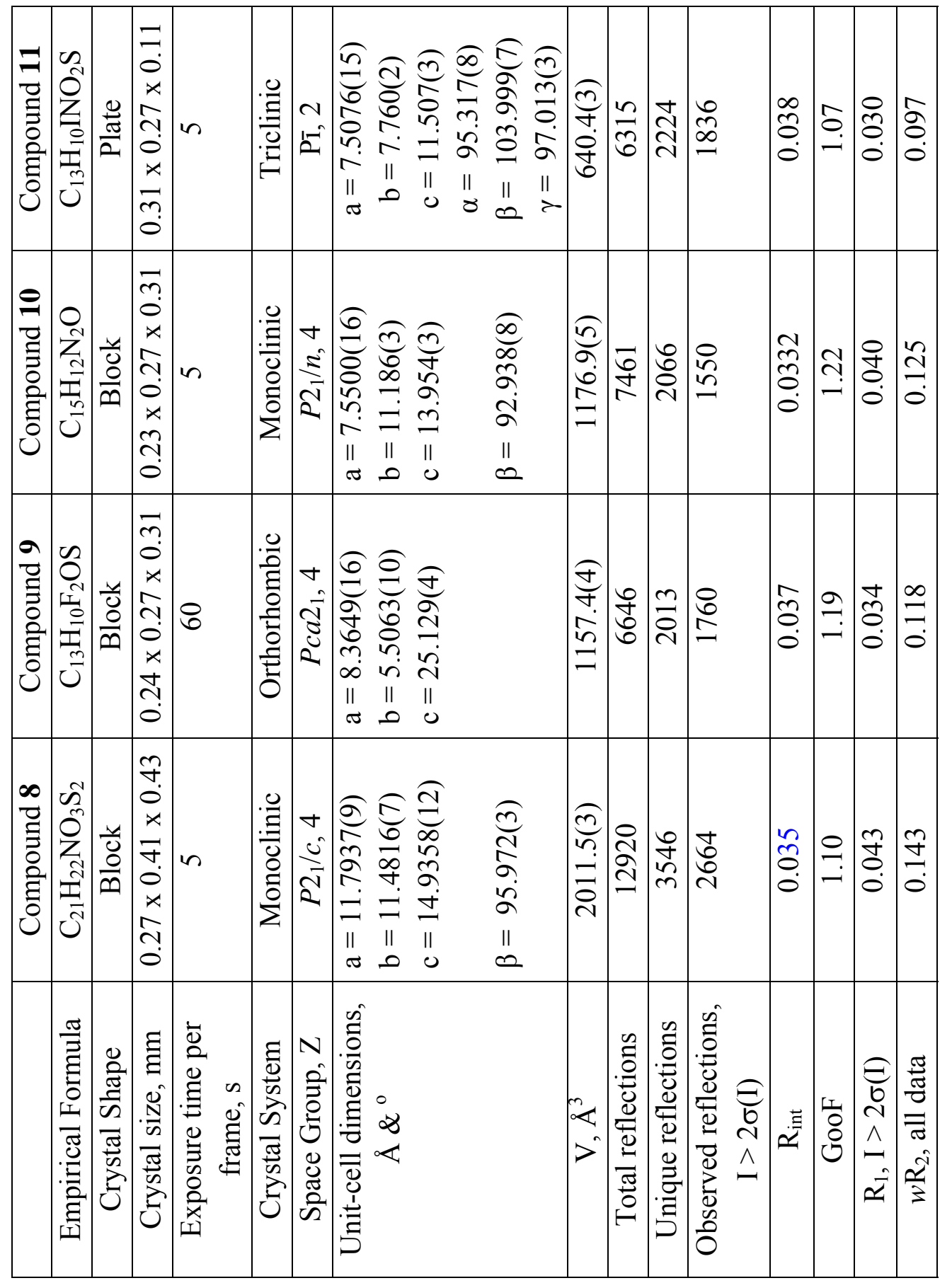




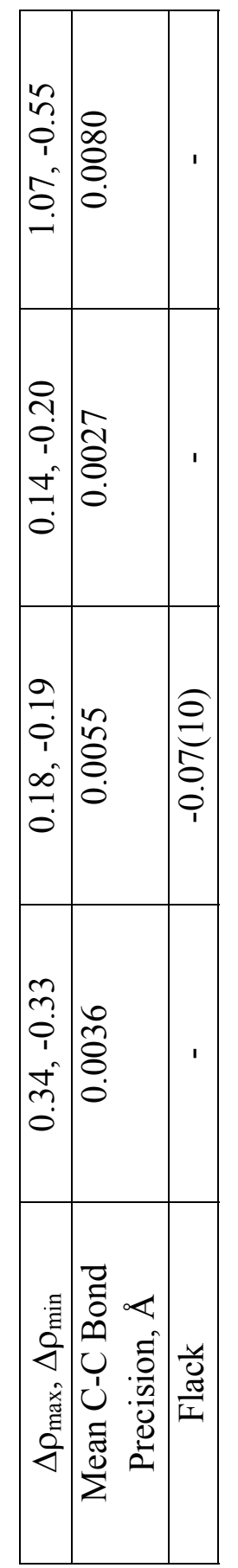


Table 4: Crystallography data obtained for compounds $12-14$.

\begin{tabular}{|c|c|c|c|}
\hline & Compound 12 & Compound 13 & Compound 14 \\
\hline Empirical Formula & $\mathrm{C}_{7} \mathrm{H}_{5} \mathrm{NO}_{3} \mathrm{~S}$ & $\mathrm{C}_{6} \mathrm{H}_{5} \mathrm{IN}_{2} \mathrm{O}_{2}$ & $\mathrm{C}_{15} \mathrm{H}_{14} \mathrm{~N}_{2} \mathrm{O}_{2} \mathrm{~S}_{1}$ \\
\hline Crystal Shape & Plate & Block & Block \\
\hline Crystal size, $\mathrm{mm}$ & $0.11 \times 0.25 \times 0.32$ & $0.40 \times 0.45 \times 0.45$ & $0.21 \times 0.31 \times 0.33$ \\
\hline $\begin{array}{l}\text { Exposure time per } \\
\text { frame, } s\end{array}$ & 10 & 5 & 10 \\
\hline Crystal System & Monoclinic & Monoclinic & Orthorhombic \\
\hline Space Group, $Z$ & $P 2{ }_{1} / c, 4$ & $P 2_{1} / c, 8$ & $P 2_{1} 2_{1} 2_{1}, 4$ \\
\hline $\begin{array}{c}\text { Unit-cell dimensions, } \\
\AA \&^{\circ}\end{array}$ & $\begin{array}{l}a=9.6083(16) \\
b=6.9347(10) \\
c=11.882(2) \\
\beta=103.834(6)\end{array}$ & $\begin{array}{l}a=7.7617(15) \\
b=12.912(3) \\
c=15.663(3) \\
\beta=95.141(6)\end{array}$ & $\begin{array}{l}a=7.7592(10) \\
b=9.9677(13) \\
c=18.230(2)\end{array}$ \\
\hline$V, \AA^{3}$ & $768.7(2)$ & $1563.5(6)$ & $1409.9(3)$ \\
\hline Total reflections & 4799 & 9857 & 8816 \\
\hline Unique reflections & 1346 & 2799 & 2458 \\
\hline Obs. reflns, $I>2 \sigma(I)$ & 1025 & 2259 & 1984 \\
\hline $\mathrm{R}_{\text {int }}$ & 0.035 & 0.030 & 0.041 \\
\hline \multicolumn{4}{|l|}{ Autostructure ${ }^{\mathrm{TM}}$} \\
\hline GooF & 1.08 & 1.04 & 1.14 \\
\hline$R 1, I>2 \sigma(I)$ & 0.059 & 0.033 & 0.040 \\
\hline$w R 2$, all data & 0.197 & 0.104 & 0.113 \\
\hline$\Delta \rho_{\max }, \Delta \rho_{\min }$ & $0.59,-0.47$ & $0.96,-0.76$ & $0.15,-0.27$ \\
\hline Flack & - & - & $0.43(12)$ \\
\hline $\begin{array}{c}\text { Mean C-C Bond } \\
\text { Precision, } \AA\end{array}$ & 0.0061 & 0.0087 & 0.0047 \\
\hline \multicolumn{4}{|l|}{ Manual Refinement } \\
\hline GooF & 1.17 & 1.02 & 1.05 \\
\hline$R 1, I>2 \sigma(I)$ & 0.039 & 0.026 & 0.037 \\
\hline$w R 2$, all data & 0.138 & 0.056 & 0.081 \\
\hline$\Delta \rho_{\max }, \Delta \rho_{\min }$ & $0.24,-0.31$ & $0.83,-0.65$ & $0.18,-0.20$ \\
\hline Flack & - & - & $0.43(8)$ \\
\hline $\begin{array}{c}\text { Mean C-C Bond } \\
\text { Precision, } \AA\end{array}$ & 0.0039 & 0.0050 & 0.0037 \\
\hline
\end{tabular}


Table 5: Crystallographic data obtained for compound 15 - 17 .

\begin{tabular}{|c|c|c|c|}
\hline & Compound 15 & Compound 16 & Compound 17 \\
\hline Empirical Formula & $\mathrm{C}_{17} \mathrm{H}_{23} \mathrm{~N}_{3} \mathrm{O}_{5} \mathrm{~S}_{2}$ & $\mathrm{C}_{7} \mathrm{H}_{11} \mathrm{~N}_{4} \mathrm{O}_{3} \mathrm{~S}$ & $\mathrm{C}_{25} \mathrm{H}_{22} \mathrm{NOPS}$ \\
\hline Crystal Shape & Block & Plate & Plate \\
\hline Crystal size, $\mathrm{mm}$ & $0.20 \times 0.30 \times 0.50$ & $0.12 \times 0.12 \times 0.32$ & $0.10 \times 0.21 \times 0.45$ \\
\hline $\begin{array}{l}\text { Exposure time per } \\
\text { frame, } s\end{array}$ & 5 & 10 & 60 \\
\hline Crystal System & Monoclinic & Monoclinic & Triclinic \\
\hline Space Group, $Z$ & $P 2_{1} / n, 4$ & $P 2_{1}, 2$ & $P \overline{1}, 2$ \\
\hline $\begin{array}{c}\text { Unit-cell dimensions, } \\
\AA \&^{\circ}\end{array}$ & $\begin{array}{l}a=7.811(4) \\
b=17.503(10) \\
c=15.892(9) \\
\beta=103.305(17)\end{array}$ & $\begin{array}{l}a=5.6245(17) \\
b=7.354(3) \\
c=12.521(5) \\
\beta=93.445(13)\end{array}$ & $\begin{array}{l}a=10.2699(10) \\
b=11.1605(10) \\
c=11.1911(12) \\
\alpha=85.945(3) \\
\beta=67.750(3) \\
\gamma=70.671(3)\end{array}$ \\
\hline$V, \AA^{3}$ & $2114(2)$ & $517.0(3)$ & $1118.07(19)$ \\
\hline Total reflections & 19952 & 3381 & 11064 \\
\hline Unique reflections & 3694 & 1776 & 3902 \\
\hline $\begin{array}{l}\text { Observed reflections, } \\
\qquad I>2 \sigma(I)\end{array}$ & 2806 & 1427 & 3000 \\
\hline$R_{\text {int }}$ & 0.044 & 0.039 & 0.035 \\
\hline GooF & 1.12 & 1.00 & 1.01 \\
\hline$R 1, I>2 \sigma(I)$ & 0.045 & 0.046 & 0.040 \\
\hline$w R 2$, all data & 0.142 & 0.092 & 0.146 \\
\hline Flack & - & $-0.25(12)$ & - \\
\hline$\Delta \rho_{\max }, \Delta \rho_{\min }$ & $0.36,-0.28$ & $0.24,-0.28$ & $0.27,-0.29$ \\
\hline $\begin{array}{c}\text { Mean C-C Bond } \\
\text { Precision, } \AA \\
\end{array}$ & 0.0041 & 0.0050 & 0.0043 \\
\hline
\end{tabular}


Table 6: Comparison of crystallography data obtained from the SMART X2S and an APEX DUO for compounds 2, 8, 12 and 16.

\begin{tabular}{|c|c|c|c|c|}
\hline & $\begin{array}{l}\text { Compound } 2 \\
\text { SMART X2S }\end{array}$ & $\begin{array}{l}\text { Compound } 2 \\
\text { APEX DUO }\end{array}$ & $\begin{array}{l}\text { Compound } 8 \\
\text { SMART X2S }\end{array}$ & $\begin{array}{l}\text { Compound } 8 \\
\text { APEX DUO }\end{array}$ \\
\hline $\begin{array}{c}\text { Unit-cell dimensions, } \\
\AA \&^{\circ}\end{array}$ & $\begin{array}{l}a=10.1375(11) \\
b=11.3871(14) \\
c=11.3412(14) \\
\beta=97.482(4)\end{array}$ & $\begin{array}{l}a=10.100(2) \\
b=11.365(2) \\
c=11.300(2) \\
\beta=97.464(5)\end{array}$ & $\begin{array}{l}a=11.7937(9) \\
b=11.4816(7) \\
c=14.9358(11) \\
\beta=95.972(3)\end{array}$ & $\begin{array}{l}a=11.7771(7) \\
b=11.4544(6) \\
c=14.9031(9) \\
\beta=95.9450(10)\end{array}$ \\
\hline$V, \AA^{3}$ & $1298.0(3)$ & $1286.1(4)$ & $2011.5(3)$ & $1999.6(2)$ \\
\hline Detector distance, $\mathrm{mm}$ & 39 & 37 & 39 & 40 \\
\hline $\begin{array}{l}\text { Exposure time per } \\
\text { frame, } \mathrm{s}\end{array}$ & 60 & 10 & 5 & 5 \\
\hline Resolution, $\AA$ & 0.84 & 0.85 & 0.84 & 0.84 \\
\hline Total time, $\mathrm{h}$ & 11.57 & 5.20 & 2.06 & 5.10 \\
\hline Total reflections & 8248 & 13491 & 12920 & 26025 \\
\hline Unique reflections & 2275 & 2180 & 3546 & 3551 \\
\hline $\begin{array}{l}\text { Observed reflections, } \\
\qquad I>2 \sigma(I)\end{array}$ & 1692 & 1698 & 2664 & 3109 \\
\hline$R_{\text {int }}$ & 0.043 & 0.039 & 0.035 & 0.024 \\
\hline GooF & 1.09 & 1.03 & 1.10 & 1.03 \\
\hline$R 1, I>2 \sigma(I)$ & 0.038 & 0.033 & 0.043 & 0.031 \\
\hline$w R 2$, all data & 0.122 & 0.085 & 0.143 & 0.085 \\
\hline$\Delta \rho_{\max }, \Delta \rho_{\min }$ & $0.23,-0.23$ & $0.14,-0.27$ & $0.34,-0.33$ & $0.30,-0.27$ \\
\hline $\begin{array}{c}\text { Mean C-C Bond } \\
\text { Precision, } \AA\end{array}$ & 0.0039 & 0.0032 & 0.0036 & 0.0025 \\
\hline
\end{tabular}

\begin{tabular}{|c|c|c|c|c|}
\hline & $\begin{array}{l}\text { Compound } \mathbf{1 2} \\
\text { SMART X2S }\end{array}$ & $\begin{array}{l}\text { Compound } 12 \\
\text { APEX DUO }\end{array}$ & $\begin{array}{l}\text { Compound 16 } \\
\text { SMART X2S }\end{array}$ & $\begin{array}{c}\text { Compound } 16 \\
\text { APEX DUO }\end{array}$ \\
\hline $\begin{array}{l}\text { Unit-cell dimensions, } \\
\AA \AA \&^{\circ}\end{array}$ & $\begin{array}{l}a=9.6083(16) \\
b=6.9347(10) \\
c=11.882(2) \\
\beta=103.834(6)\end{array}$ & $\begin{array}{l}a=9.5830(12) \\
b=6.9252(8) \\
c=11.8518(15) \\
\beta=103.815(3)\end{array}$ & $\begin{array}{l}a=5.6245(17) \\
b=7.354(3) \\
c=12.521(5) \\
\beta=93.445(13)\end{array}$ & $\begin{array}{l}a=5.6095(10) \\
b=7.3299(15) \\
c=12.491(2) \\
\beta=93.297(5)\end{array}$ \\
\hline$V, \AA^{3}$ & $768.7(2)$ & $763.78(16)$ & $517.0(3)$ & $512.74(17)$ \\
\hline Detector distance, $\mathrm{mm}$ & 39 & 38 & 39 & 38 \\
\hline $\begin{array}{l}\text { Exposure time per } \\
\text { frame, } \mathrm{s}\end{array}$ & 5 & 5 & 10 & 10 \\
\hline Resolution, $\AA$ & 0.84 & 0.82 & 0.84 & 0.84 \\
\hline Total time, $\mathrm{h}$ & 2.06 & 2.33 & 3.40 & 3.27 \\
\hline
\end{tabular}




\begin{tabular}{|c|c|c|c|c|}
\hline Total reflections & 4799 & 5166 & 3381 & 3017 \\
\hline Unique reflections & 1346 & 1473 & 1776 & 1940 \\
\hline $\begin{array}{c}\text { Observed reflections, } \\
I>2 \sigma(I)\end{array}$ & 1025 & 1283 & 1427 & 1825 \\
\hline$R_{\text {int }}$ & 0.035 & 0.022 & 0.039 & 0.019 \\
\hline GooF & 1.17 & 1.06 & 1.00 & 1.13 \\
\hline$R 1, I>2 \sigma(I)$ & 0.039 & 0.031 & 0.046 & 0.031 \\
\hline$w R 2$, all data & 0.138 & 0.081 & 0.092 & 0.073 \\
\hline$\Delta \rho_{\max }, \Delta \rho_{\min }$ & $0.24,-0.31$ & $0.28,-0.31$ & $0.24,-0.28$ & $0.21,-0.22$ \\
\hline $\begin{array}{c}\text { Mean C-C Bond } \\
\text { Precision, } \AA\end{array}$ & 0.0039 & 0.0026 & 0.0050 & 0.0030 \\
\hline Flack & - & - & $-0.25(12)$ & $-0.02(8)$ \\
\hline
\end{tabular}


Table 7: Comparison of crystallography data obtained from the SMART X2S and an APEX DUO for compounds 11 and 18 (4-methyl- $N$-phenyl-benzenesulfonamide).

\begin{tabular}{|c|c|c|c|c|}
\hline & $\begin{array}{l}\text { Compound } 11 \\
\text { SMART X2S }\end{array}$ & $\begin{array}{l}\text { Compound } 11 \\
\text { APEX DUO }\end{array}$ & $\begin{array}{l}\text { Compound } \mathbf{1 8} \\
\text { SMART X2S }\end{array}$ & $\begin{array}{l}\text { Compound } \mathbf{1 8} \\
\text { APEX DUO }\end{array}$ \\
\hline Empirical Formula & $\mathrm{C}_{13} \mathrm{H}_{10} \mathrm{INO}_{2} \mathrm{~S}$ & $\mathrm{C}_{13} \mathrm{H}_{10} \mathrm{INO}_{2} \mathrm{~S}$ & $\mathrm{C}_{13} \mathrm{H}_{12} \mathrm{NO}_{2} \mathrm{~S}$ & $\mathrm{C}_{13} \mathrm{H}_{12} \mathrm{NO}_{2} \mathrm{~S}$ \\
\hline Crystal size, $\mathrm{mm}$ & $0.11 \times 0.17 \times 0.31$ & $0.11 \times 0.17 \times 0.31$ & $0.18 \times 0.30 \times 0.32$ & $0.18 \times 0.30 \times 0.32$ \\
\hline $\begin{array}{c}\text { Exposure time per } \\
\text { frame, } s\end{array}$ & 5 & 5 & 60 & 10 \\
\hline Detector Distance & 39 & 37 & 39 & 37 \\
\hline $\begin{array}{c}\text { Unit-cell } \\
\text { dimensions, } \\
\AA \&^{\circ}\end{array}$ & $\begin{array}{l}a=7.5076(15) \\
b=7.760(2) \\
c=11.507(3) \\
\alpha=95.317(8) \\
\beta=103.999(7) \\
\gamma=97.013(3)\end{array}$ & $\begin{array}{l}a=7.4946(11) \\
b=7.7421(11) \\
c=11.4816(16) \\
\alpha=95.418(3) \\
\beta=103.879(3) \\
\gamma=96.985(3)\end{array}$ & $\begin{array}{l}a=10.2890(11) \\
b=11.0563(12) \\
c=13.4402(15) \\
\alpha=67.823(3) \\
\beta=87.573(4) \\
\gamma=67.616(3)\end{array}$ & $\begin{array}{l}a=10.259(3) \\
b=11.050(4) \\
c=13.380(5) \\
\alpha=67.808(8) \\
\beta=87.291(8) \\
\gamma=67.435(7)\end{array}$ \\
\hline$V, \AA^{3}$ & $640.4(3)$ & $636.65(16)$ & 1299.9(2) & $1288.4(7)$ \\
\hline Total reflections & 6315 & 7653 & 12855 & 23008 \\
\hline Unique reflections & 2224 & 2786 & 4545 & 4600 \\
\hline $\begin{array}{c}\text { Obs. reflections, } \\
I>2 \sigma(I)\end{array}$ & 1836 & 2492 & 3364 & 3117 \\
\hline$R_{\text {int }}$ & 0.038 & 0.025 & 0.036 & 0.039 \\
\hline GooF & 1.07 & 1.05 & 1.01 & 1.10 \\
\hline$R 1, I>2 \sigma(I)$ & 0.030 & 0.028 & 0.055 & 0.062 \\
\hline$w R_{2}$, all data & 0.097 & 0.066 & 0.174 & 0.266 \\
\hline$\Delta \rho_{\max }, \Delta \rho_{\min }$ & $1.07,-0.55$ & $1.41,-0.43$ & $0.25,-0.40$ & $0.37,-0.44$ \\
\hline $\begin{array}{c}\text { Mean C-C Bond } \\
\text { Precision, } \AA\end{array}$ & 0.0055 & 0.0045 & 0.0059 & 0.0079 \\
\hline
\end{tabular}

